\title{
GRUPO OPERATIVO: estratégia educativa no cuidado ao paciente diabético com
}

\author{
obesidade
}

OPERATING GROUP: educational strategy in care of obesity diabetic patient

GRUPO OPERATIVO: estrategia educativa en la atención del paciente diabético con obesidad

Recebido: 16/04/2021 | Revisado: 23/04/2021 |Aceito: 26/04/2021 | Publicado: 10/05/2021

Ananda Santos Freitas
ORCID: https://orcid.org/0000-0002-6420-3945
Universidade Estadual do Maranhão, Brasil
E-mail: annandhacx@ @otmail.com
Andressa Vieira Freitas
ORCID: https://orcid.org/0000-0002-2169-2689
Centro Universitário Santo Agostinho, Brasil
E-mail: andressa-maia17@ hotmail.com
Jéssica Barroso de Moraes
ORCID: https://orcid.org/0000-0002-2450-0240
Eentro Universitário de Ciências e Tecnologia do Maranhão, Brasil
E-mail: jessimoraes2017.2@hotmail.com
Kameny Santos Franco
ORCID: https://orcid.org/0000-0001-6056-3663
Centro Universitário Santo Agostinho, Brasil
E-mail: kamenyfranco@gmail.com
Pammela Weryka da Silva Santos
ORCID: https://orcid.org/0000-0002-9931-0951
Universidade Federal do Piauí, Brasil
E-mail: pammela_weryka @hotmail.com
Raiany Kayre Pereira Salomão
ORCID: https://orcid.org/0000-0003-4328-7008
E-mail: raianykayre@hotmail.com
Marisa Araujo Costa
ORCID: https://orcid.org/0000-0002-8312-1697
Universidade Estadual do Maranhão, Brasil
E-mail: josaeneide.tc@gmail.com

\begin{abstract}
Resumo
O estudo teve como objetivo relatar a experiência de um grupo operativo com diabéticos obesos de uma estratégia de saúde da família. Trata-se de uma pesquisa do tipo relato de experiência, realizada com pacientes diabéticos obesos de uma Unidade Básica de Saúde da cidade de Caxias, Maranhão, Brasil, no período de maio a agosto de 2019. Participaram 10 usuários portadores do DM tipo 2, do sexo feminino, da faixa etária entre 37 a 76 anos e diagnosticados com obesidade grau I, II e III. Foram realizados cinco encontros com uma hora de duração, com temas relacionados à saúde e a qualidade de vida. As temáticas abordadas nos grupos operativos foram: Conhecendo a condição crônica; Abordagem sobre o que é estilo de vida; Integralidade e multidimensionalidade da saúde; Autocuidado e autonomia e Plano de vida. Os grupos operativos possibilitaram a participação ativa de todos. Assim, estes grupos foram direcionados aos diabéticos obesos que buscavam mudanças de comportamento. A interação grupal promoveu um ambiente oportuno, livre de preconceitos e estigmas, que motivou os diabéticos a mudar o estilo de vida, sobretudo, no que diz respeito, ao controle do peso corporal.
\end{abstract}

Palavras-chave: Diabetes; Obesidade; Educação em saúde.

\section{Abstract}

The present study aimed to report the experience of an operative group with obese diabetics of a family health strategy. This is an experience report, conducted with obese diabetic patients of a Basic Health Unit (UBS) in the city 
of Caxias, Maranhão, Brazil, from May to August 2019. Ten users with type 2 DM, female, aged 37 to 76 years and diagnosed with grade I, II and III obesity participated of the activities. Five one-hour meetings were held on topics related to health and quality of life. The themes addressed in the operating groups were: Knowing the chronic condition; Approach to what is lifestyle; Integrality and multidimensionality of health; Self-care and autonomy and Life plan. The operating groups enabled the active participation of all. Thus, these groups were directed to obese diabetics who sought behavioral changes. The group interaction promoted an opportune environment, free of prejudice and stigmas, which motivated diabetics to change their lifestyle, especially with regard to body weight control.

Keywords: Diabetes; Obesity; Health education.

\section{Resumen}

El estudio tuvo como objetivo reportar la experiencia de un grupo operatorio con diabéticos obesos en una estrategia de salud familiar. Se trata de una encuesta tipo informe de experiencia realizada con pacientes obesos diabéticos en una Unidad Básica de Salud de la ciudad de Caxias, Maranhão, Brasil, de mayo a agosto de 2019. Participaron 10 usuarios con DM tipo 2, mujeres, con edades entre 37 y 76 años y diagnosticado con obesidad grado I, II y III. Se realizaron reuniones de cinco horas de duración sobre temas relacionados con la salud y la calidad de vida. Los temas abordados en los grupos operativos fueron: Conocer la condición crónica; Aproximación a lo que es estilo de vida; Integralidad y multidimensionalidad de la salud; Autocuidado y autonomía y Plan de Vida. Los grupos operativos permitieron la participación activa de todos. Por lo tanto, estos grupos estaban dirigidos a diabéticos obesos que buscaban cambios de comportamiento. La interacción grupal promovió un entorno oportuno, libre de prejuicios y estigmas, que motivó a los diabéticos a cambiar su estilo de vida, especialmente en lo que respecta al control del peso corporal.

Palabras clave: Diabetes; Obesidad; Educación para la salud.

\section{Introdução}

O diabetes mellitus (DM) é definido como um grupo heterogêneo de distúrbios metabólicos que apresenta em comum à hiperglicemia, resultantes de defeitos na ação da insulina, na secreção de insulina ou ambas. Sua classificação compreende quatro classes clínicas: DM tipo 1 (DM1), DM tipo 2 (DM2), outros tipos específicos de DM e DM gestacional (Sociedade Brasileira de Diabetes, 2015).

O aumento na prevalência do DM deve-se a fatores como o crescimento e envelhecimento populacional, o processo de urbanização, a globalização de maus hábitos alimentares, e a crescente prevalência da obesidade e sedentarismo (Vitoi et al., 2015). Ademais, existe forte associação do DM com a problemática da obesidade, pois a maioria dos pacientes com esta doença apresenta excesso de peso (Wilding, 2014).

Esta associação possui grande relevância contemporânea por ser o DM um dos problemas mundiais de saúde mais importantes da atualidade, em vista de sua elevada prevalência, morbidade e mortalidade (Monteiro et al., 2015). A obesidade, por sua vez, também é vista como problema de saúde pública, sobretudo nos países em desenvolvimento, que apresentam 115 milhões de pessoas acima do peso (Vinholes \& Pacheco, 2015).

Diante das complicações ou comorbidades inerentes do DM que podem interferir no processo de autocuidado, a educação em saúde surge como uma estratégia de promoção da saúde, na medida em que se busca capacitar o portador de DM com obesidade a problematizar sobre sua condição, através do incentivo a mudanças do estilo de vida e corresponsabilidade por seu processo de saúde-doença. Deste modo, o apoio educacional, por meio das tecnologias em saúde, pode produzir um impacto positivo sobre o comportamento das pessoas com diabetes e outras doenças associadas (Kewming et al., 2016).

Em meio às novas tecnologias para o manejo das Doenças Crônicas Não Transmissíveis (DCNT), ressalta-se o Grupo Operativo (GO), que vem sendo utilizado em diversas áreas do Sistema Único de Saúde (SUS), principalmente na Atenção Primária à Saúde (APS). Esses grupos podem ser empregados para promoção da saúde, prevenção de doenças e prestação de cuidados específicos aos participantes. Seus benefícios incluem: maior otimização do trabalho, diminuição das consultas individuais, participação ativa do indivíduo no processo educativo e envolvimento da equipe de profissionais com o paciente (Almeida \& Soares, 2010; Costa et al., 2016; Menezes \& Avelino, 2016). 
Portanto, é indispensável que os profissionais da saúde discutam e aprendam sobre os fenômenos grupais, pois a organização desses grupos como modalidade de atenção coletiva é cada vez mais frequente em nosso sistema de saúde, principalmente como prática educativa e preventiva na APS. Neste sentido, o presente estudo teve como objetivo relatar a experiência de um grupo operativo com diabéticos obesos de uma Estratégia de Saúde da Família (ESF).

\section{Metodologia}

Trata-se de uma pesquisa do tipo relato de experiência, com abordagem de natureza qualitativa. Segundo Pereira, Shitsuka, Parreira (2018) estudos qualitativos, são aqueles nos quais é importante a interpretação por parte do pesquisador com suas opiniões sobre o fenômeno em estudo. Neles a coleta de dados muitas vezes ocorre por meio de entrevistas com questões abertas, na qual o pesquisador é o principal instrumento.

O estudo foi realizado no período de maio a agosto de 2019 com diabéticos obesos de uma Unidade Básica de Saúde (UBS) da cidade de Caxias, Maranhão, Brasil. Os pacientes foram selecionados em dois momentos: no primeiro, realizou-se uma triagem pelo nutricionista com todos os portadores de DM (137) da UBS, para avaliar as necessidades de atendimentos dos pacientes, a presença de fatores de risco para o desenvolvimento de complicações e o controle glicêmico. Por meio da triagem, identificou-se que 24 eram obesos, destes, dezesseis tinham obesidade grau I, cinco obesidade grau II e três obesidade grau III. Diante disto, selecionaram-se 10 diabéticos com as seguintes características: aqueles com os maiores Índices de Massa Corporal (IMC) e Circunferência Abdominal (CA), independente do grau de obesidade; os que haviam sido acometidos por outras enfermidades como Hipertensão Arterial Sistêmica (HAS) e Acidente Vascular Encefálico (AVE); sedentários e aqueles com hábitos alimentares inadequados.

Após a seleção, os pacientes foram convidados a participar do grupo juntamente com seus respectivos acompanhantes ou cuidadores. Os usuários que aceitaram participar das atividades eram portadores do DM tipo 2, do sexo feminino, da faixa etária entre 37 a 76 anos e diagnosticados com obesidade grau I, II e III.

De acordo com Mendes (2012), os GOs são compostos por seus integrantes, um coordenador e um observador. Os integrantes devem ser no máximo 15 pessoas, o que justifica o número de participantes da presente pesquisa. Esses integrantes precisam afiliar-se, ao GO, pela identificação com os objetivos do grupo, que neste caso, diz respeito ao controle do peso dos pacientes diabéticos.

Os encontros dos grupos ocorreram em uma sala privada, climatizada e silenciosa. Os encontros aconteceram quinzenalmente, o que correspondeu a um total de cinco encontros, com duração de 60 minutos cada. Todos os GOs contaram com a participação de sete a dez pacientes. O coordenador, observador e os facilitadores eram enfermeiras, nutricionista, farmacêutica e fisioterapeutas, integrantes da Residência Multiprofissional em Saúde da Família, assim como o preceptor da residência.

Como estratégia pedagógica e educativa, buscou-se promover a mudança do estilo de vida dos diabéticos obesos, através da utilização do GO. Os grupos desenvolvidos foram registrados em livro ata do serviço, contendo o facilitador da atividade, número de participantes, seus dados antropométricos e nutricionais, a temática abordada, os questionamentos feitos pelos usuários, data e duração do grupo.

No que tange à condução do GO, inicialmente houve a apresentação de cada participante, coordenador, observador e facilitadores. Em seguida, o objetivo do grupo foi exposto, e os participantes, questionados sobre suas dificuldades para o controle do sobrepeso e obesidade. É importante ressaltar que o profissional coordenador era responsável por garantir a comunicação entre os integrantes do GO, o observador por registrar e desenvolver uma percepção global dos encontros e o facilitador por conduzir e aplicar a atividade educativa. Essas definições de papéis dentro do GO proporcionaram um momento 
seguro para que todos aprendessem uns com os outros, compartilhassem suas dificuldades e desejos para mudança do estilo de vida.

A organização dos dados foi realizada mediante o levantamento dos temas emergentes do grupo e foram apresentados na ordem sequencial em que apareceram. Os conteúdos manifestados foram analisados e discutidos à luz da literatura científica.

\section{Resultados}

\section{$1^{0}$ Encontro: Conhecendo a condição crônica}

No que diz respeito ao GO "Conhecendo a condição crônica", os assuntos trabalhados detalharam os princípios básicos da educação em saúde ao diabético, isto é, a função metabólica das pessoas com e sem diabetes, fatores de risco e complicações em longo prazo, mitos e verdades sobre o DM e como controlar os sinais e sintomas da doença. O GO comparou o corpo de um indivíduo saudável ao do diabético utilizando uma bola como modelo para representação de uma célula, com vistas à compreensão do usuário.

Neste primeiro contato, ocorreu a avaliação nutricional destes usuários realizada pela nutricionista da equipe. Na ocasião os diabéticos passaram por uma avaliação das medidas antropométricas como peso, altura, circunferência da cintura. Durante a consulta de nutrição, foram prescritas metas referentes à mudança dos hábitos alimentares, visto que, a redução do consumo de alimentos inadequados foi a principal dificuldade relatada pelos participantes, tais metas, deveriam ser alcançadas até o próximo GO.

Durante o encontro, evidenciou-se que a maioria dos convidados possuíam conhecimento sobre o DM, suas complicações e como evitá-las, porém, a dificuldade para mudança do estilo de vida, no que tange aos hábitos alimentares e a prática de atividade física, foi referida por mais da metade dos diabéticos, o que pode ser atribuída à presença da obesidade como uma complicação apresentada por eles. Além disso, este primeiro GO esclareceu dúvidas e favoreceu a troca de experiência entre os pacientes que se confortaram uns com os outros.

\section{$2^{\circ}$ Encontro: Abordagem sobre estilo de vida}

O GO "Abordagem sobre estilo de vida" teve como objetivos: promover uma reflexão sobre a importância da reformulação dos hábitos atuais nocivos à saúde como alimentação inadequada, sedentarismo, estresse, e o de estimular as práticas de relaxamento ou alternativas que beneficiem a saúde física, mental e social.

Deste modo, os tópicos discutidos foram à alimentação saudável, prática de atividade física, qualidade do sono e estresse. Os facilitadores demonstraram por meio de material expositivo as consequências dos comportamentos inadequados para a saúde, bem como os benefícios do consumo de alimentos saudáveis e os efeitos positivos do exercício físico.

Durante os relatos e as trocas de experiências no grupo, foi evidenciada a existência de dúvidas no que diz respeito aos alimentos que o indivíduo diabético poderia consumir, a quantidade e qualidade destes. Neste momento, a nutricionista realizou uma nova avaliação dos pacientes, a partir dos dados antropométricos mensurados no dia e dos questionamentos feitos no GO. As metas nutricionais foram avaliadas e um novo planejamento das refeições e estratégias para uma alimentação saudável foi efetuado.

Quanto à atividade física, um número significativo dos usuários referiu praticá-la, o que tornou o grupo dinâmico, participativo e com maior troca de vivências, agindo como incentivo para que os sedentários iniciassem ou retomassem o exercício físico. Os sedentários obtiveram orientações da fisioterapeuta quanto a prática do exercício de modo correto e a mesma lançou metas a esses pacientes para que inserissem o exercício gradativamente no dia a dia. 


\section{$3^{\circ}$ Encontro: Integralidade e multidimensionalidade da saúde}

O terceiro encontro teve o intuito de abordar a saúde na sua integralidade e multidimensionalidade. Para isso, promoveu-se uma autoanálise dos integrantes em relação aos seus hábitos de vida dentro do contexto da integralidade do indivíduo e suas escolhas. O conteúdo foi explanado através de uma palestra promovida por uma das enfermeiras, que utilizou de imagens ilustrativas para abordagem do tema. A metodologia e os recursos empregados foram elaborados a fim de causar um impacto nos participantes, no que se refere ao controle inadequado do diabetes e ao surgimento de comorbidades.

Os pacientes relataram seus sentimentos, medos, angústias, dúvidas acerca das consequências que o DM e a obesidade podem ocasionar. Discutiu-se sobre o propósito que cada um pretende alcançar participando do GO e sobre o nível de confiança para controlar o peso corporal pela adoção de um novo estilo de vida. O monitoramento do peso realizado em cada encontro tem sido fundamental para a adesão dos usuários ao GO. Portanto, todos os envolvidos no grupo apresentaram redução significativa no peso e circunferência da cintura, fator este relacionado ao alcance das metas nutricionais e à prática de atividade física.

\section{$4^{\circ}$ Encontro: Autocuidado e autonomia}

$\mathrm{O}$ quarto GO objetivou estimular o amadurecimento dos conceitos de autocuidado, autoestima, autonomia e corresponsabilização pelos cuidados com a saúde. Para isso o penúltimo encontro contou com a participação de alguns profissionais convidados pela equipe de idealizadores do grupo. Na oportunidade, ocorreu uma roda de conversa com uma fisioterapeuta residente em Atenção ao Câncer da cidade de São Paulo. Durante a explanação, relatou as experiências no seu local de trabalho, a importância do autocuidado, da autoestima, da adesão aos tratamentos e da autonomia para a tomada de decisões.

Posteriormente, uma podóloga palestrou sobre o diabetes e o cuidado com os pés. O mediador em sua abordagem lúdica demonstrou a forma correta de visualização dos pés para identificação de sinais de alerta, como calosidades, pele desidratada e diminuição da sensibilidade do membro. Os pacientes foram orientados sobre os cuidados no domicílio e sobre o momento de procurar atendimento de saúde. Durante a discussão do tema, o grupo compartilhou experiências e cada um dos participantes recebeu uma avaliação dos pés pela profissional, o que possibilitou ao diabético aprender sobre os cuidados com os pés por meio de situações cotidianas como: o uso de sapatos e meias adequadas, uso do filtro solar no membro, higiene e cuidados com as unhas.

Ao longo do GO, evidenciou-se que alguns dos indivíduos desconheciam as práticas apresentadas e compartilhadas, porém já haviam vivenciado em algum momento situações de risco, como uso inadequado de calçados, formações de calosidades e sua evolução para ferimentos. Ademais, os mesmos passaram por uma consulta com a nutricionista que readequou a dieta dos pacientes que não alcançaram uma modificação efetiva do peso corporal e forneceu novas metas para os que progrediram significativamente no objetivo. A frequência de atividade física também foi avaliada e adaptada individualmente pela fisioterapeuta.

\section{$5^{\circ}$ Encontro: Plano de vida}

O último GO teve como objetivo relacionar os elementos-chave na proposta de construção de um plano e projeto de vida, como forma de promover uma análise das qualidades pessoais dos participantes a partir de suas próprias histórias. Para isso, foram discutidos tópicos referentes à definição conceitual de plano e projeto de vida, elaboração e análise de planilhas de mudança de hábitos de vida e avaliação dos encontros.

O facilitador da atividade utilizou uma planilha composta pelos aspectos pessoais da roda da vida que incluem: amigos e familiares, lazer, vida financeira, intelecto, espiritualidade, amor, trabalho/carreira e saúde. Os pacientes teriam que 
atribuir pontuações ( 0 a 10) a cada um destes aspectos e, posteriormente, traçar metas ou objetivos para aqueles que obtivessem menor pontuação. Os relacionados ao intelecto, lazer e vida financeira foram menos pontuados pelos entrevistados, que relataram os motivos para as notas obtidas, dentre eles: a não realização de atividades que promovam momentos de lazer e relaxamento, a falta de investimento em sua educação e de um rendimento financeiro suficiente para suprir as necessidades básicas.

Este momento permitiu identificar os pontos de partida para a etapa de ação do plano de vida e para orientar estes pacientes acerca dos aspectos essenciais para uma mudança de comportamento mais efetiva. Os profissionais repassaram informações cruciais para que estes indivíduos seguissem as recomendações trabalhadas ao longo dos encontros, de modo a não desistirem dos objetivos ali alcançados.

Além disso, neste último encontro, uma nova avaliação do peso e da prática de atividade física foi realizada. A mesma constatou que os participantes cumpriram as metas repassadas nos GOs, modificaram os hábitos alimentares e o estilo de vida sedentário, o que repercutiu positivamente no controle do peso corporal. Pacientes anteriormente diagnosticados com obesidade grau I, II e III alcançaram redução do grau da enfermidade.

Os diabéticos avaliaram a proposta do $\mathrm{GO}$ e além de satisfeitos com os resultados obtidos, mostraram-se agradecidos pelo trabalho desenvolvido pela equipe da UBS, sendo estes convidados a participarem das oficinas de autocuidado que serão futuramente promovidas à população acometida por doenças crônicas.

\section{Discussão}

A educação em saúde é um conjunto de saberes e práticas voltadas para a promoção da saúde e prevenção de doenças. Neste contexto, pode-se mencionar o GO que possibilita trabalhar com a população diversos temas que se considere importante para uma formação em saúde mais reflexiva, integrada e humanizada (Lucchese et al., 2013).

Seguindo este pensar, evidencia-se que as atividades realizadas no estudo proporcionaram mudanças no cotidiano desses pacientes, o que pode ser atribuído ao tipo de abordagem utilizada, uma vez que esta provocou maior sensibilização da população através de motivações e informações, que devem ser transferidas de maneira efetiva para que ocorra a educação em saúde.

Almeida e Soares (2010) identificaram, ao trabalhar com diabéticos por meio do GO, uma maior interação entre os participantes, o fortalecimento do diálogo e da comunicação em saúde, que reforçou o processo de aprendizagem. Estes achados corroboram com o da presente pesquisa, pois observou-se que a apresentação de temáticas referentes ao controle do DM e a obesidade no formato de GO, resultou em um grupo dinâmico, participativo e com maior troca de vivências. Os resultados também demonstraram mudança na atitude dos usuários, quanto à aquisição de autonomia para o autocuidado e manejo correto da condição.

A tecnologia do GO foi também utilizada por Oliveira et al. (2016), para a aprendizagem do cuidado por mães de filhos com deficiência. Os resultados da presente pesquisa mostraram que os princípios do grupo operativo se fizeram presentes na experiência das mães que dele participaram. O contexto grupal foi expresso como um espaço agenciador de práticas de acolhimento, vínculo e escuta, pois privilegiou em sua abordagem, o compartilhamento dos desafios enfrentados pelas participantes.

Diante desses estudos percebe-se que os grupos voltados para a educação em saúde funcionam como estratégias eficientes, os quais abrem espaços para a escuta das necessidades das pessoas e para aquisição de informação. Os GOs permitem que todos possam falar sobre seus problemas e buscar soluções, conjuntamente com os profissionais, tendo em vista um objetivo em comum, como é o caso do controle do peso por diabéticos obesos (Lucchese et al., 2014). 
O grupo operativo é reconhecido por facilitar a expressão de seus participantes, acerca do que pensam, sentem, garantindo o seu bem-estar individual. O compartilhamento de vivências possibilita reflexões entre os envolvidos, o que contribui, por exemplo, para a acolhida da temática proposta pelos coordenadores do grupo (Nascimento \& Galindo, 2017).

Estas práticas grupais no cotidiano da Atenção Básica (AB), como ferramenta de promoção da saúde, integram a lista de reorientação dos serviços proposta pelo Ministério da Saúde (MS), uma vez que, fazem parte dos procedimentos financiados pelo SUS, por serem consideradas ferramentas que ampliam o conhecimento do usuário sobre seus problemas, e favorecem mudanças nos hábitos de vida que representam risco a saúde (Nogueira et al., 2016)

Através da realização dos GOs, os usuários alcançaram redução significativa dos maus hábitos alimentares, do sedentarismo, bem como do peso corporal. Esses objetivos foram conquistados, graças à tecnologia em saúde utilizada, que trouxe como benefícios: um ambiente seguro isento de estigma ou preconceito, que permitiu conhecer os desejos e anseios dos participantes que concluíram o GO com mais autonomia e adesão a novos comportamentos. De acordo com Hoffmann e Máximo (2019), isso foi possível, por ser o GO um encontro que se solidariza o refletir e o agir de seus integrantes e não somente um ato de depositar ideias de um sujeito no outro.

Além disso, as ações educativas em saúde podem capacitar pessoas e grupos na construção de novos saberes, conduzindo a uma prática consciente de comportamentos preventivos ou de promoção da saúde. Essas atividades, expandem as possibilidades de controle das doenças, de reabilitação e tomada de decisões, que contribuem para uma vida mais saudável. Tal processo é facilmente conquistado pela utilização da técnica de grupos operativos (Carvalho et al., 2020).

Há equipes que se baseiam em outras técnicas metodológicas de grupo que não possuem embasamento teórico e ainda assim alcançam resultados. No entanto, o grupo operativo destaca-se por ser uma ferramenta de incorporação do saber caracterizada pela didática horizontal, que torna o indivíduo um agente ativo e responsável pela mudança de hábitos (Menezes \& Avelino, 2016).

Outras experiências costumam trabalhar apenas com atividades educativas e nomear de GO, reduzindo essa nova ferramenta de trabalho a uma roda de conversa ou a um bate-papo. Percebe-se um uso descontextualizado de termos sem articulação com seus respectivos fundamentos, o que compromete os resultados terapêuticos associados a essa modalidade de grupo (Silva et al., 2018).

Deste modo, ressalta-se que a proposta do GO como estratégia educativa perpassa tais associações, pois este, possibilita a construção coletiva de projetos, fruto do diálogo e da escuta do espaço grupal, em busca da execução de tarefas em comum entre seus participantes. Ademais, o GO que historicamente, foi projetado para uso na saúde mental, ganhou espaço em outras áreas da saúde, por meio da condução dos processos de mudanças de hábitos de vida, como por exemplo, entre aqueles que convivem com doenças crônicas transmissíveis como o diabetes (Lima et al., 2014).

\section{Conclusão}

A realização do grupo operativo com diabéticos obesos permitiu identificar o comportamento que os usuários apresentavam em relação à própria doença. Os pacientes também demonstraram maior interesse pelos relatos de vida dos demais participantes, bem como pelos temas apresentados pelos profissionais, o que resultou em um aprendizado sociocultural entre os envolvidos e em uma maior aproximação da equipe de saúde.

Além disso, abordaram-se temáticas relevantes para o autocuidado do paciente diabético, como o surgimento da doença, o tratamento, as complicações, alimentação saudável, prática de exercício físico, cuidados com os pés, autoestima, dentre outros. A interação grupal promoveu um ambiente oportuno, livre de preconceitos e estigmas, que motivou os envolvidos a mudar o estilo de vida, sobretudo, no que diz respeito, ao controle do peso corporal. Os pacientes foram 
convidados para participar dos novos grupos como agentes multiplicadores tendo em vista, a adesão de novos participantes, pois a experiência com o GO será ampliada aos demais diabéticos da unidade.

Deste modo, é de extrema importância a utilização do GO como tecnologia de manejo das condições crônicas, pois tem impacto positivo sobre as formas de autocuidado do paciente, uma vez que, torna o usuário protagonista e corresponsável pelo seu processo de saúde-doença.

Ademais, o grupo operativo constitui uma relevante estratégia educacional que pode ser utilizada em vários contextos da atenção primária em saúde, por estar centrado na aprendizagem e na transformação. Essa experiência pode ser ainda, implantada nos demais serviços de saúde, especialmente em situações de formação de recursos humanos em saúde. Contudo, esta tecnologia pode ser evidenciada em algumas pesquisas como complexa e promissora, e ainda pouco utilizada. Neste sentido, sugere-se a realização de estudos sobre a temática, e sobre seu posicionamento metodológico para que seja utilizada de forma correta e como fonte de conhecimento para pesquisas futuras.

\section{Referências}

Almeida, S. P., \& Soares, S. M. (2010). Aprendizagem em grupo operativo de diabetes: uma abordagem etnográfica. Cienc. Saúde Colet.,15, $1123-1132$.

Carvalho, M. R., de Sá, A. N. P., de Morais, J. D., Gomes, A. C., de Farias, D. N., \& de Lima, L. M. M. (2020). Atuação da fisioterapia em grupo operativo na Atenção Básica. Revista de Educação Popular, 144-159.

Costa, N. P. et al (2016). Storytelling: a care technology in continuing education for active ageing. Rev. Bras. Enferm., 69(6), 1132-1139.

Hoffmann, J., \& Máximo, C. E. (2019). A Educação Popular em Saúde como dispositivo transformador das práticas de produção da saúde no município de Itajaí-SC. Revista Pesquisas e Práticas Psicossociais, 14(1), 1-14.

Kewming, S., D’amore, A., \& Mitchell, E. K. L. (2016). Conversation Maps and Diabetes Education Groups: An Evaluation at an Australian Rural Health Service. Diabetes Spectr, 29(1), 32-36.

Lima, M. G., Ceccato, M. D. G. B., de Souza Braga, D., da Silva, F. M. B., Gonçalves, M. A., de Melo Gajo, M., \& Mendes, D. F. (2014). Grupos operativos de hipertensos e diabéticos no pet-saúde. Revista Brasileira de Pesquisa em Saúde, 16(1), 133-138.

Lucchese, R., Vargas, L. S., Teodoro, W. R., Santana, L. K. B., \& Santana, F. R. (2013). Tecnologia de grupo operativo aplicada ao programa de controle do tabagismo. Texto Contexto Enferm, 22 (4), 918-26.

Lucchese, R., Vera, I., Benicio, P. R., da Silva, A. D. F., Munari, D. B., \& Fortuna, C. M. (2014). Uso do grupo operativo na atenção em saúde: revisão integrativa. Cogitare Enfermagem, 19(4), 823-832.

Mendes, E. V. (2012). O cuidado das condições crônicas na atenção primária à saúde: o imperativo da consolidação da estratégia da saúde da família. Brasília: Organização Pan-Americana da Saúde.

Menezes, K. K. P., \& Avelino, P. R. (2016). Grupos operativos na Atenção Primária à Saúde como prática de discussão e educação: uma revisão. Cad. Saúde Colet., 24(1), 124-130.

Monteiro, C. N. et al (2015). Cobertura de serviços públicos de saúde para gastos com medicamentos e vacinas na população com diabetes mellitus. Ciênc. Saude Colet., 20 (2), 557-564.

Nascimento, T. M., \& Galindo, W. C. M. (2017). Grupo Operativo em Centros de Atenção Psicossocial na opinião de psicólog as. Revista Pesquisas e Práticas Psicossociais, 12(2), 422-438.

Nogueira, A. L. G., Munari, D. B., Fortuna, C. M., \& Santos, L. F. (2016). Pistas para potencializar grupos na Atenção Primária à Saúde. Revista Brasileira de Enfermagem, 69(5), 964-971

Oliveira, D. M. et al (2016). O grupo operativo como instrumento de aprendizagem do cuidado por mães de filhos com deficiência. Esc. Anna Nery, 20(3).

Pereira, A. S., Shitsuka, D. M., Parreira, F. J., \& Shitsuka, R. (2018). Metodologia da pesquisa científica. [e-Book]. Santa Maria. Ed. UAB / NTE / UFSM.

Silva, M. A. M., Marques, F. M., Brito, M. D. C. C., Viana, R. S., Mesquita, A. L. M., Silva, A. S. R., \& Gomes, L. C. (2018). Grupo operativo com primigestas: uma estratégia de promoção à saúde. Revista Brasileira em Promoção da Saúde, 31(1).

Sociedade Brasileira de Diabetes (2015). Diretrizes da Sociedade Brasileira de Diabetes 2014-2015. AC Farmacêutica.

Vinholes, D. B., \& Pacheco, H. A. (2015). Perfil do risco cardiovascular de pacientes diabéticos atendidos em ambulatório de especialidades. Ciên Saúde, $7(3), 116-122$.

Vitoi, N. C. et al (2015). Prevalência e fatores associados ao diabetes em idosos no município de Viçosa, Minas Gerais. Rev. Bras. Epidemiol., 18(4), 953-965.

Wilding, J. P. (2014). The importance of weight management in type 2 diabetes mellitus. Int J Clin Pract., 68(6),682-691. 